Revista Española de Antropología Americana

ISSN: 0556-6533

https://dx.doi.org/10.5209/reaa.72827

\title{
Sinopsis de la arqueología de Tierra del Fuego
}

\author{
Martín Vázquez ${ }^{1}$ y Luis A. Borrero²
}

Recibido: 2 de diciembre 2020 / Aceptado: 7 de enero 2021

Resumen. La arqueología del Archipiélago Fueguino puede entenderse como un caso de variadas trayectorias culturales que se ajustan a la notable diversidad ambiental existente. Se introducen y comentan aquí los resultados principales alcanzados por las investigaciones arqueológicas realizadas a lo largo del tiempo en los distintos sectores de la Isla Grande de Tierra del Fuego y sus alrededores. Las escasas evidencias antiguas, de poco más de 10.000 años, refieren a cazadores-recolectores que aún interactuaban con fauna extinta. Durante el Holoceno Temprano y la primera parte del Holoceno Medio, la señal arqueológica regional se hace algo más intensa en algunos sectores como la costa norte del canal Beagle y algunos segmentos de la costa Atlántica y el estrecho de Magallanes. Los sitios fechados hacia el Holoceno Tardío se hacen progresivamente más abundantes en toda la región. Especialmente durante los últimos 1500 años, cuando se registra una mayor intensidad en el uso de los distintos ambientes y se incluyen áreas con escasa o nula ocupación previa dentro de los circuitos de movilidad de cazadores terrestres y litorales.

Palabras clave: arqueología; Tierra del Fuego; cazadores-recolectores-pescadores.

\section{[en] Synopsis of the Archaeology of Tierra del Fuego}

\begin{abstract}
The archaeology of the Fuegian archipelago can be understood as an example of diverse cultural trajectories related with corresponding high environmental diversity. The main archaeological results of the different areas of the archipelago are introduced and commented. The limited evidences of the initial occupations, older than 10,000 years, inform about terrestrial hunter-gatherers still interacting with extinct fauna. In the north coast of the Beagle channel, segments of the Atlantic coast and the Strait of Magellan the regional archaeological signal intensifies during the early Holocene and the first part of the middle Holocene. Sites dated during the Late Holocene are progressively more abundant everywhere, especially during the last 1500 years, when there is increasing intensity of use of the different environments and when new areas are included within the mobility circuits of both terrestrial and littoral hunter-gatherers.
\end{abstract}

Keywords: Archaeology; Tierra del Fuego; Hunter-Gatherer-Fishers.

Sumario: 1. Introducción. 2. Beyond Here Lies Nothin': las ocupaciones tempranas del Pleistoceno Final-Holoceno Temprano. 3. The Times Are A-Changin: la transición Holoceno Temprano-Holoceno Medio. 4. Ancient footprints are everywhere: Holoceno Tardío. 5. Palabras finales. 6. Referencias.

Cómo citar: Vázquez, Martín y Luis A. Borrero. 2021. «Sinopsis de la arqueología de Tierra del Fuego». Revista Española de Antropología Americana 51: 173-185.

\footnotetext{
CADIC-CONICET. IDEI-UNTdF. vazquezmartin68@gmail.com.

IMHICIHU-CONICET. Facultad de Filosofía y Letras, Universidad de Buenos Aires. laborrero2014@gmail. com.
} 


\section{Introducción}

El archipiélago fueguino se ubica en el extremo sur de la Patagonia, de la cual está separada por el estrecho de Magallanes. La isla principal y de mayor extensión es la Isla Grande de Tierra del Fuego, la cual presenta un sector estepario al norte (Figuras 1 y 2a), una faja central boscosa y hacia el sur se extiende la parte más austral de la cordillera de los Andes. A lo largo del borde occidental y meridional del archipiélago se proyecta un gran arco de canales e islas que se extiende desde al Pacífico hasta el Atlántico y tiene como vértice sur el cabo de Hornos (ver Figura 1, Figura 3a).

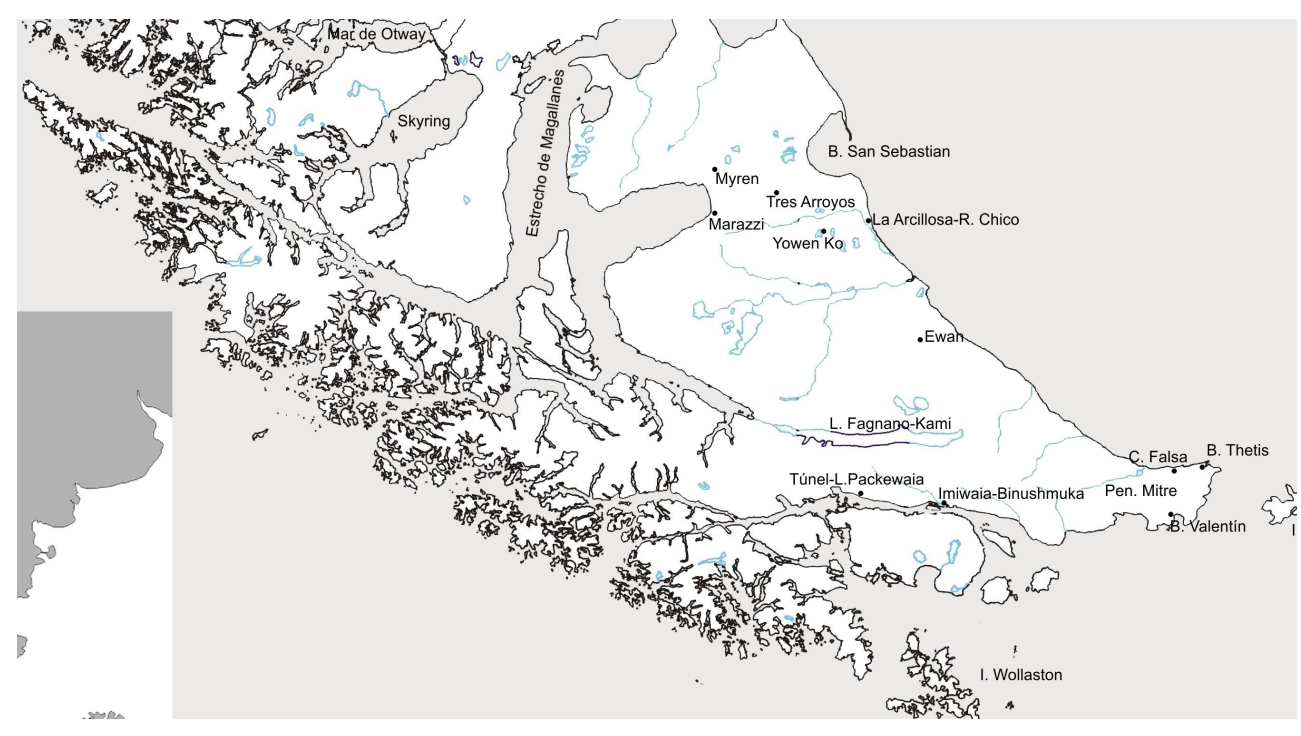

Figura 1. Mapa de ubicación con los principales sitios mencionados en el texto.

Desde hace más de un siglo los pueblos originarios fueguinos ocupan un espacio destacado en el pensamiento antropológico, habiendo sido invocados como análogos de las primeras etapas del poblamiento americano, e incluso de la que alguna vez se llamó "Edad de Piedra" del Viejo Mundo. Sin embargo, no fue hasta fines de la década de 1970 que el establecimiento de programas de investigación permitió articular un corpus de información sobre las trayectorias histórico-evolutivas de estas sociedades. En este capítulo exponemos un breve resumen de los resultados de la investigación alcanzados en las últimas décadas, reseñando las principales tendencias expresadas en el registro arqueológico de Tierra del Fuego.

\section{Beyond Here Lies Nothin': las ocupaciones tempranas del Pleistoceno Final- Holoceno Temprano}

En Tierra del Fuego, durante el lapso $11000-8000^{3}$ años AP la señal arqueológica es extremadamente baja. Existe un único sitio del Pleistoceno Final, el alero Tres Arro-

Las fechas presentadas en este trabajo se refieren a años radiocarbónicos sin calibrar. 
yos 1 en el Cerro de los Onas (Figura 2b). Sus ocupaciones más antiguas están datadas aproximadamente entre 10685 y 9960 AP. Cinco fogones informan acerca de unas pocas visitas al sitio, repetidas probablemente en lapsos cortos. Se trata de cazadores que básicamente han consumido camélidos y caballo americano (Hippidion) (Borrero 2003; Massone 2004, 2009). La recuperación de huesos de Rheidae datados en 9960 AP constituyó la primera evidencia para la presencia de esta ave no voladora en la isla (Martin et al. 2009). Debe destacarse que las ocupaciones en este sitio son esencialmente similares en contenido y edad a aquellas registradas en el sur del continente en Cueva Fell, Cueva del Medio y Cueva Lago Sofía 1. Esto sugiere que estas localizaciones habrían formado parte de un circuito frecuentado de manera recurrente por estas tempranas poblaciones, en tiempos anteriores a la formación del estrecho de Magallanes.
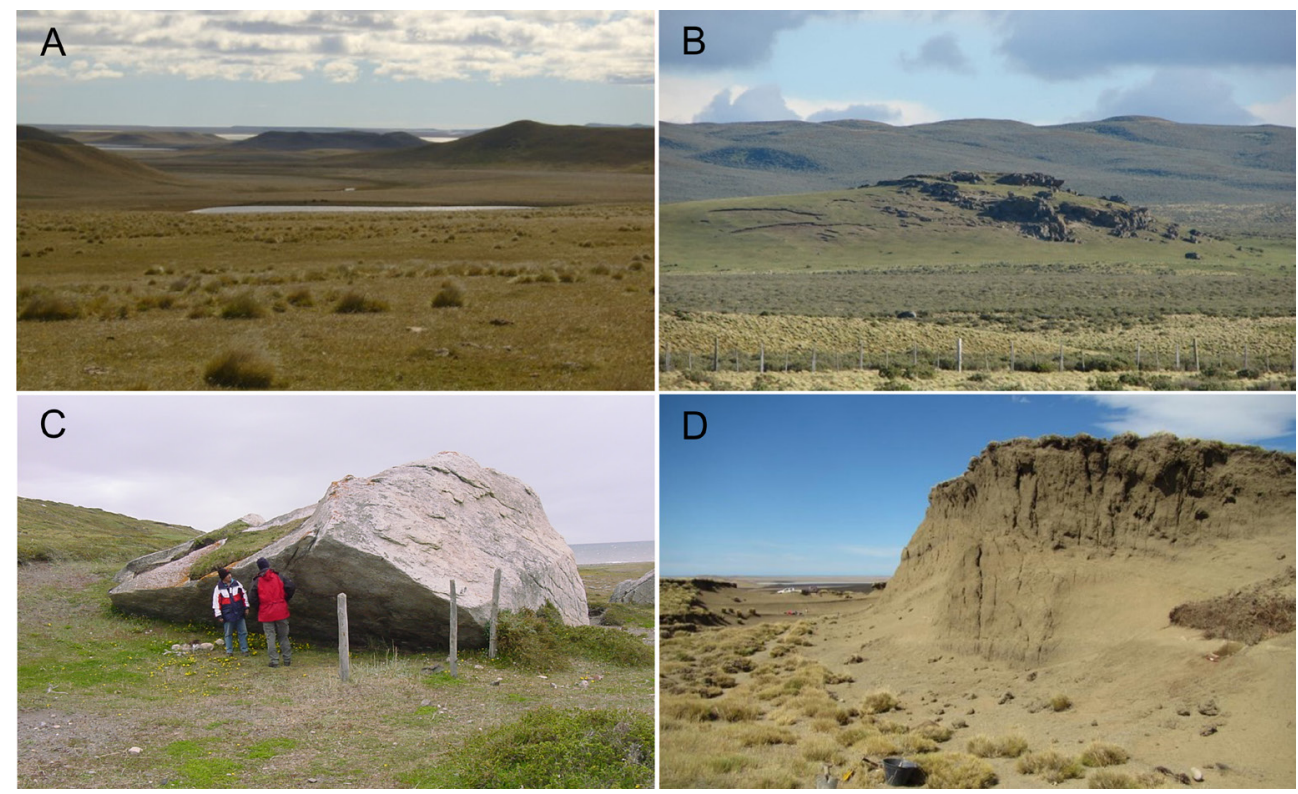

Figura 2. a) Paisaje de la estepa en el norte de Tierra del Fuego; b) Cerro de los Onas, donde se emplaza el alero Tres Arroyos; c: Sitio Marazzi 1; d) Sitio Yowen Ko.

\section{The Times Are A-Changin': la transición Holoceno Temprano-Holoceno Medio}

El que sigue es un tiempo en el que los archipiélagos fueguinos están experimentando una serie de modificaciones sustanciales, fundamentalmente relacionadas con el ascenso del nivel del mar, unido a componentes isostáticos y tectónicos (Isla y Bujalesky 2008). Los niveles marinos sufrieron un incremento progresivo desde fines del Último Máximo Glaciar, hasta alcanzar cotas equivalentes a las actuales hacia los 8000 años AP (Ponce et al. 2011; Montes y Martinioni 2017). La inundación de la artesa glaciar que hoy ocupa el Estrecho de Magallanes y la consecuente desaparición del puente terrestre con el continente habría ocurrido entre los $c a .8200$ y 7500 años AP (McCulloch y Morello 2009). Los niveles marinos continuaron en ascenso por aproximadamente dos milenios, alcanzando sus máximas cotas entre los 6000 
y 5000 AP, para luego descender paulatinamente hasta su posición actual hacia los 4500/4000 AP (entre otros, Bujalesky 1998; Rabassa et al. 2000). Estas trasformaciones estuvieron acompañadas por una tendencia general al mejoramiento del clima y la expansión del bosque de Nothofagus, que se expresa a escala regional en perfiles polínicos y otros proxies (McCulloch et al. 2000; Rabassa et al. 2000; Montes y Martinioni 2017). En este escenario de transformaciones climáticas y geográficas se registran nuevas evidencias en la costa norte del canal Beagle. Éstas provienen de tres sitios: Túnel I, Imiwaia I y Binushmuka I (ver Figura 1), con fechados que las sitúan dentro del lapso 7900 y los 6900 años AP (Orquera y Piana 1999; Piana et al. 2012; Zangrando et al. 2018).

Estos contextos, Primer Componente de Túnel I y Capa S de Imiwaia, a diferencia de los componentes posteriores, no consisten en depósitos conchíferos. Tradicionalmente fueron asociados a grupos de cazadores terrestres que habrían incursionado sobre estas costas y sin vinculación evidente con los grupos canoeros que los sucederían (Orquera y Piana 1999). Si bien estos grupos podrían haber aprovechado recursos litorales en algún grado -las condiciones marinas ya estaban establecidas en el Beagle desde al menos los 7900 años AP, y se hallaron unos pocos restos de pinnípedos en el Primer Componente de Túnel I-, nada hacía pensar en algún tipo de adaptación litoral o vinculación con los pueblos canoeros que poco tiempo después dominarían el paisaje cultural regional. Sin embargo, la evidencia procedente de Binushmuka ha permitido evaluar alternativas a estas hipótesis previas, sugiriendo una posible vinculación o continuidad entre estas ocupaciones y las posteriores. Zangrando y colaboradores han introducido el concepto de "Early Coastal Foragers" para englobar a las sociedades que ocuparon las costas del Beagle entre los $7900 \mathrm{y}$ los 6900 AP (Zangrando et al. 2018).

La calibración de los fechados radiocarbónicos disponibles indican que sólo poco más de dos siglos separan estos conjuntos de la aparición de sociedades con subsistencias orientadas a la explotación de recursos marinos y movilidad náutica (Zangrando et al. 2018). Los testimonios más tempranos de estos pueblos fueron localizados en distintos puntos del Archipiélago Occidental Patagónico, desde Chiloé hasta el Cabo de Hornos, y los fechados más antiguos se colocan entre los 6400-6000 años $\mathrm{AP}^{4}$. Los yacimientos se caracterizan por la formación de concheros y por el énfasis en la explotación de recursos litorales (Orquera y Piana 1999, 2006).

Algunos de estos componentes presentan una considerable extensión temporal, como por ejemplo los casos del Segundo Componente de Túnel I o las capas K, L y M de Imiwaia I (Figura 3b), cuyas ocupaciones se suceden por más de dos milenios (Orquera y Piana 1988, 1999; Zangrando et al. 2018). A los mencionados se pueden sumar otros con cronologías distribuidas entre los 6000 y los 4000 años AP, como el Componente Antiguo de Lancha Packewaia, la Capa F de Mischiwen, además de las ocupaciones más antiguas del Sitio 11 de bahía Valentín (Figura 3d) -costa sur de Península Mitre-, para las cuales se han señalado similitudes con el canal Beagle (Zangrando et al. 2009).

4 En la región del Archipiélago Magallánico Fueguino -además de los que se mencionan para la región del Beagle- los sitios más representativos de la primera colonización de estos pueblos son: Grandi I y Bayly, al sur de Isla Navarino; Bahía Buena y Punta Santa Ana, sobre la margen occidental del estrecho de Magallanes (Legoupil et al. 2011; San Román 2014, 2016); Skyring y Engefield en los mares interiores de Otway y Skyring (Legoupil 1997, 2000; San Román et al. 2002; San Román 2016). 


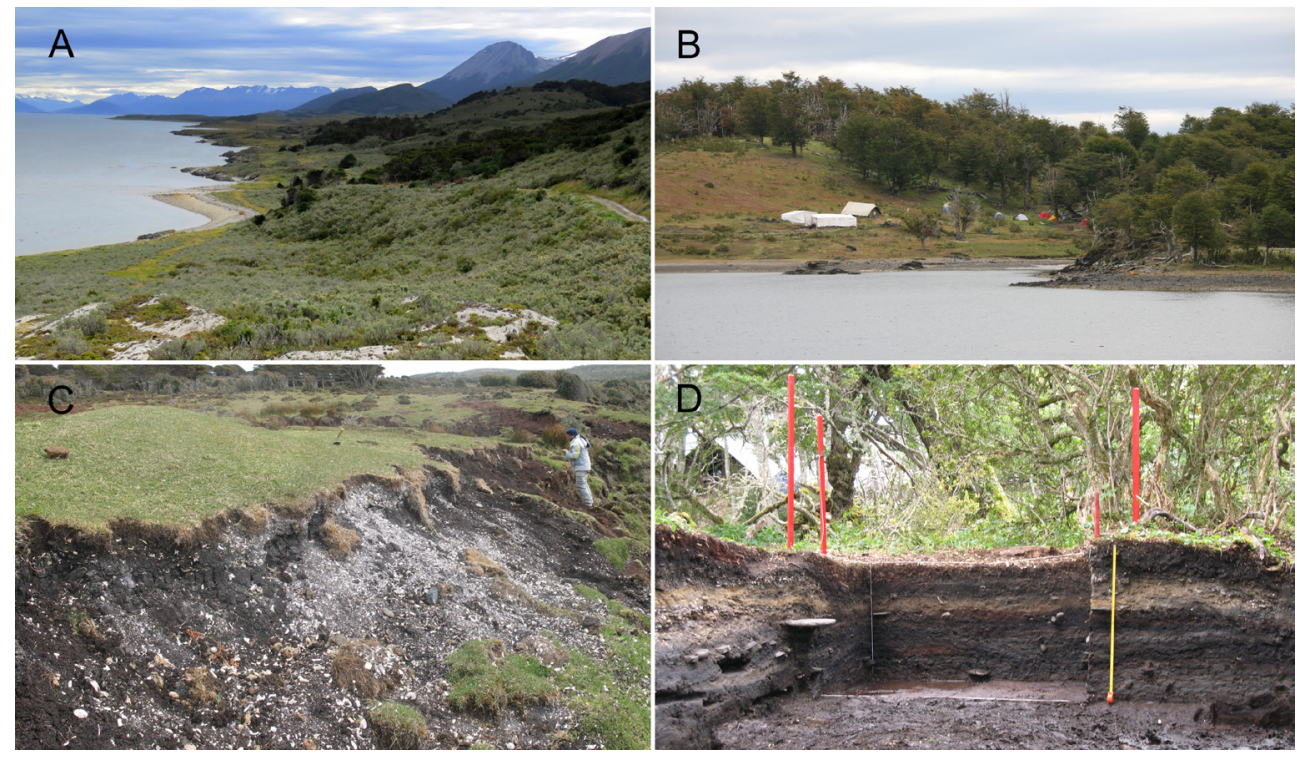

Figura 3. a) Paisaje de la costa norte del Canal Beagle; b) Sitio Imiwaia I; c) Domo de conchero en la localidad bahía Thetis; d) Excavación del sitio Bahía Valentín 11.

Orquera y Piana han englobado a estas poblaciones en lo que denominaron "Tradición Adaptativa de los canales Magallánico-Fueguinos”, y que luego ampliaron haciéndola extensible a todo el litoral sudoccidental sudamericano, desde Chiloe hasta el Cabo de Hornos (Orquera y Piana 1988, 2006). Esta "Tradición Cultural” fue definida por el aprovechamiento intensivo de recursos litorales y de espacios marinos adyacentes, con un uso complementario de recursos terrestres; el desarrollo de medios instrumentales específicos para la explotación de estos recursos y la movilidad: arpones de punta separable y canoas en este caso (Orquera y Piana 1988, 1999, 2006). La gran mayoría de estos sitios son concheros y se ha señalado el uso recurrente de materias primas de origen litoral para la confección de sus equipamientos, con una sofisticada tecnología ósea que incluye arpones, punzones y cuñas (Scheinsohn 1993-1994; Orquera y Piana 1999; Christensen 2016; San Román 2016). Otro rasgo característico es la presencia de obsidiana verde (Morello et al. 2004), procedente de la zona del mar de Otway, lo que sumado a la existencia de similitudes en los patrones decorativos de arpones y otros artefactos óseos de ambas regiones llevó a proponer la existencia de una dinámica poblacional que propició la existencia de redes de circulación de personas, bienes e ideas a escala macroregional (Fiore 1999; Álvarez 2014).

Como se mencionó, para el norte y centro de Tierra del Fuego no se conocen contextos datados para la primera parte del Holoceno Medio. Recién hacia los 5000 AP se registran evidencias de ocupaciones en la costa del estrecho de Magallanes -en los sitios Marazzi 1 (Figura 2c) y Cabo Monmouth 20-, en la costa Atlántica -en la localidad La Arcillosa y los sitios Cerro Bandurrias y Río Chico $1^{5}$, y hacia el inte-

\footnotetext{
Los casos mencionados para la costa atlántica presentan depósitos de tipo conchero. Por el contrario, Marazzi 1 y Cabo Monmouth 20, sobre la costa del estrecho de Magallanes, no presentan este tipo de depósitos, aunque sí se registra la presencia de recursos de origen marino.
} 
rior de la estepa -en sitios como Myren 2 y los contextos 1 y 2 del sitio Yowen Ko (Figura 2d)-(Morello et al. 1999; Salemme et al. 2007a; Morello et al. 2012; Calás 2014; Oría y Mari 2019). En todos estos casos se trata de sitios a cielo abierto o, en el caso de Marazzi 1, de un alero muy expuesto. Todas estas manifestaciones incluyen variados repertorios de instrumentos y recursos consumidos, muchos de los cuales indican adaptaciones a los nuevos hábitats isleños. Algunos de los materiales son interpretables como resultado de divergencia cultural, con respecto a ancestros más antiguos, relacionada con el aislamiento de poblaciones en la isla (Borrero 19891990; Charlin et al. 2013; Scheinsohn 2014).

\section{Ancient footprints are everywhere: Holoceno Tardío}

En distintas zonas del archipiélago fueguino se constata notable amplificación de la señal arqueológica hacia el Holoceno Tardío, y más aun dentro de los últimos 1500 años. Es innegable que, cuanto mayor es la antigüedad de los yacimientos, éstos han estado más tiempo expuestos a procesos erosivos o acresionales, lo que disminuye sus probabilidades de preservación y detección por parte de los arqueólogos. Sin embargo, parece difícil defender que el notable aumento de la señal arqueológica regional se deba sólo a un proceso de destrucción de yacimientos antiguos o de obstrusividad diferencial. Esta tendencia está acompañada por el crecimiento en la variabilidad de sitios, la ocupación de nuevos espacios y configuraciones ambientales, la aparición de inhumaciones, la incorporación de nuevos tipos de artefactos y armas, así como la modificación de antiguos diseños y el abandono o la incorporación de algunos tipos de materias primas, como las del chorrillo Miraflores (Borrazzo et al. $2019)^{6}$. Estas transformaciones se fueron sucediendo con distintos ritmos de aparición en las distintas regiones del archipiélago.

Los cazadores que habitaron en el interior del norte de la isla enfocaban su subsistencia en proteína de mamíferos terrestres, pero -aunque los conjuntos están dominados por restos de fauna terrestre- las dietas siempre incluían proporciones pequeñas de elementos marítimos (Barberena 2004).

Los sitios en el norte se multiplican durante el Holoceno Tardío. En Tres Arroyos, por ejemplo, tras unos 9000 años de abandono, no sólo el alero es intensivamente ocupado por cazadores de guanaco desde $c a$. 1400 AP (Muñoz 1997; Massone 2004), sino que se identificaron numerosas ocupaciones en otros lugares del Cerro de los Onas (ver Figura 2b), tanto al aire libre como bajo roca. En la zona central de la costa atlántica también se registraron ocupaciones de este periodo, como por ejemplo en las localidades San Pablo y María Luisa (Borrero 1985; Lanata 1995). De hecho la mayoría de los yacimientos conocidos en el norte y centro de Tierra del Fuego están datados dentro de los últimos 2000 años radiocarbónicos (Massone et al. 1993; Salemme et al. 2007b; Borrero et al. 2008; Borrazzo 2009, 2010; Morello et al. 2012; Oría y Salemme 2019; Oría et al. 2021). Con excepción de algunas concentraciones en la costa esteparia, se trata de ocupaciones generalmente efímeras, muchas de ellas localizadas en relación con lagunas estacionales. Excepcionalmente

6 La fuente primaria de este tipo de materia prima fue localizada en el norte de Tierra del Fuego y rocas de este tipo han sido registradas en sectores tan alejados como la Península Mitre (Borrazzo 2009, 2012; Borrazzo et al. 2019). 
hay registros de notables acumulaciones óseas, como en Las Vueltas, un yacimiento interpretado como un lugar de matanza colectiva de guanacos (Santiago y Salemme 2016); otras acumulaciones óseas notables en los campos de dunas de arcilla de la bahía San Sebastián resumen numerosas ocupaciones superpuestas y mezcladas por la circulación de agua (Borrazzo 2010). Otros contextos con edades similares se emplazan en relación con afloramientos rocosos en el norte y centro de la Isla Grande, tal es el caso de Bloque Errático 1, Cabeza de León, San Julio1, o se concentran junto a acantilados costeros, como Punta María, entre otros (Borrero 1985). Los entierros en el norte de la isla son muy variados, desde individuos directamente en dunas o concheros (Martin 2004, 2006; Salemme et al. 2007b; Santiago et al. 2011; Prieto et al. 2020) al uso de Chenques (Ocampo et al. 2000). Todas éstas constituyen evidencias de poblaciones muy móviles. Igualmente ocurre con los sitios en los bosques del centro de la isla, cerca del lago Fagnano-Kami, los cuales tienen ocupaciones ya desde ca. 3000 AP (De Angelis 2015). Este área es la misma en que se concentraron los últimos campamentos selk'nam, precisamente en los bosques que se extienden desde la boca del río Fuego hasta las cabeceras del lago Fagnano-Kami.

Los primeros contactos con occidentales se remontan al siglo XVI, y se fueron haciendo más frecuentes en los siglos posteriores. Desde fines del XIX la instalación de misiones y estancias establece una situación de contacto que implicó dramáticas consecuencias para los pueblos originarios. El periodo estuvo marcado por la expansión de un modelo ganadero y las explotaciones loberas y auríferas. Entre los registros arqueológicos más notables podemos mencionar al sitio Ewan, en un bosque abierto al noreste del Fagnano-Kami, que incluye dos loci históricos, uno de los cuales ha sido asociado con un sitio ceremonial (Mansur y Piqué 2012) y para la misma época en las costas del Beagle, Túnel VII y Lanashuaia I (Estévez y Gassiot 2002). En estas ocupaciones, al igual que en otras tardías en distintos sectores de la isla, se utilizó intensamente el vidrio como materia prima (De Angelis 2015). La utilización de restos de naufragios en las costas de Península Mitre también ha sido registrada arqueológicamente en diversas localidades (Vázquez 2019).

Para el caso de las secuencias arqueológicas del sur de Tierra del Fuego, tanto en el canal Beagle central, como en áreas exteriores (Islas Wollaston, Península Mitre e Isla de los Estados), la tendencia indica la existencia de pocos sitios con fechados anteriores a los 4000 años y un aumento exponencial a partir de los últimos dos milenios (Orquera y Piana 1999; Vázquez et al. 2011).

En relación con la dimensión tecnológica resulta notable el abandono de la decoración en el instrumental óseo a partir de 4000 años AP (Fiore 1999) y una tendencia a una menor inversión laboral en su confección. Por su parte, luego de los ca. 4000 años AP, se incorpora la técnica bifacial, y junto con ésta los cabezales líticos en contextos del Beagle central y posteriormente cabezales líticos interpretados como puntas de flechas hacia los 1400 años AP (Álvarez 2014).

En el sur de Tierra del Fuego no se conocen entierros datados en fechas más antiguas que los 2000 años AP. Si bien no son frecuentes, en los bosques del canal Beagle se utilizaron pequeños aleros o concheros para disponer a los muertos (Piana et al. 2006; Álvarez et al. 2008). También en Península Mitre fueron registradas inhumaciones con cronologías comparables en Caleta Falsa, bahía Thetis y bahía Valentín (Figura 3d) (Tessone et al. 2011). Parece defendible que el hallazgo de restos humanos para los últimos 2000 años estaría reflejando un aumento en la complejidad 
y frecuencia de las prácticas mortuorias que no parece atribuible sólo a problemas de muestreo o sesgos tafonómicos; más bien estaría reflejando la reducción de la movilidad y un aumento demográfico a escala regional.

Por su parte, la evidencia isotópica de restos humanos procedentes del canal Beagle muestra una señal de dieta marítima, que es concordante con el contenido registrado en concheros (Tessone et al. 2003; Kochi 2017). Existe una continuidad importante de caza y recolección marítima a través del tiempo, puntuado con momentos o sectores en que los guanacos crecen en importancia (Orquera y Piana 1999; Zangrando 2009). Por ejemplo, si bien se ha sostenido la importancia de otáridos complementados por aves y peces, se han identificado incrementos en las frecuencias de restos de guanacos hacia los 5000 AP (Zangrando 2009) y los 19001000 AP, para luego mostrar una abrupta declinación durante la denominada Pequeña Edad de Hielo (Fernández et al. 2020). Para tiempos algo más recientes -ca. $1500 \mathrm{AP}$ - se ha postulado la existencia de un proceso de intensificación, expresado principalmente por el aumento de restos de peces y aves, con la consecuente disminución de restos de otáridos en las arqueofaunas del Beagle (Zangrando 2009; Tivoli 2010). Esta intensificación estuvo basada sobre el incremento de esfuerzos de estas presas dentro de un contexto en el que la aparición de vecinos pudo ser importante.

Hacia el extremo sud oriental, en Península Mitre e Isla de los Estados, el panorama se torna más complejo. En Bahía Valentin (ver Figura 3d) -emplazada en la costa sur de Península Mitre-, para cuyas ocupaciones del Holoceno Medio se señalaron algunas similitudes con las ocupaciones del canal Beagle, presenta mayor variación en las ocupaciones posteriores (Zangrando et al. 2009; Vázquez et al. 2011). Al considerar algunos contrastes entre las costas norte y sur de la Península, la arqueología de ese sector de la isla se torna aún más compleja (Vidal 1985; Lanata 1995; Muñoz y Belardi 2011; Vázquez 2019). Sumado a este panorama arqueológico, que aún debe ser estudiado con mayor profundidad, las ocupaciones de Isla de los Estados datadas entre los 2700 y 1500 años AP, que necesariamente deben de haber dependido de movilidad náutica, completan un panorama muy variable en una región relativamente pequeña (Horwitz y Weissel 2011).

\section{Palabras finales}

Los registros arqueológicos de Tierra del Fuego dan cuenta de diversas trayectorias evolutivas mostrando un continuum de adaptaciones en relación con una amplia diversidad ambiental. La principal diferenciación reconocida es que las ocupaciones identificadas al norte de la cordillera fueguina pueden considerarse mayoritariamente enfocadas en recursos terrestres, con buenos registros desde el Holoceno Medio. Por su parte, hacia la misma época, al sur de la cordillera, la evidencia arqueológica indica la irrupción de sociedades con el manejo de medios de navegación litoral y una economía enfocadas en la explotación de recursos marinos.

Tras estos variados procesos que tuvieron lugar a lo largo de miles de años, las configuraciones culturales de los últimos siglos adquirieron lentamente la fisonomía etnográfica que fuera clásicamente reconocida para los pueblos originarios de Tierra del Fuego. 


\section{Referencias}

Álvarez, Myriam R. 2014. «Caminando sobre tus huellas: tecnología lítica en sociedades cazadoras-recolectoras-pescadoras del canal Beagle», en Cazadores de mar y tierra. Estudios recientes en arqueología fueguina, Jimena Oría y Angélica Tivoli, eds., pp. 109124. Ushuaia: Editora Cultural Tierra del Fuego, Museo del Fin del Mundo.

Alvarez, Myriam R., Martin Vázquez y Ernesto Piana. 2008. «Prácticas mortuorias entre los cazadores-recolectores del canal Beagle: el caso de Shamakush entierro». Magallania 36 (2): 105-121. http://dx.doi.org/10.4067/S0718-22442008000200007.

Barberena, Ramiro. 2004. "Arqueología e isótopos estables en Tierra del Fuego», en Arqueología del Norte de la Isla Grande de Tierra del Fuego, Luis A. Borrero y Ramiro Barberena, eds., pp. 135-169. Buenos Aires: Dunken.

Borrazzo, Karen. 2009. «El uso prehistórico de los afloramientos terciarios en la bahía San Sebastián (Tierra del Fuego, Argentina)», en Arqueología de la Patagonia. Una mirada desde el último confin, Mónica Salemme, Fernando Santiago, Myriam Álvarez, Ernesto Piana, Martín Vázquez y Estela Mansur, eds., pp. 291-305. Ushuaia: Utopías.

- 2010. Arqueología de los esteparios fueguinos. Tecnología y tafonomía lítica en el norte de Tierra del Fuego. Tesis Doctoral, Facultad de Filosofía y Letras, Universidad de Buenos Aires.

-. 2012. «Raw Material Availability, Flaking Quality and Hunter-Gatherer Decision Making in Northern Tierra del Fuego Island (Argentina)». Journal of Archaeological Science 39 (8): 2643-2654. https://doi.org/10.1016/j.jas.2012.03.018

Borrazzo, Karen, Luis A. Borrero y Maria C. Pallo. 2019. «Exploring Lithic Transport in Tierra del Fuego (Southern South America)». Journal of Archaeological Science: Reports 24: 220-230.

Borrero, Luis A. 1985. La economía prehistórica de los habitantes del norte de la Isla Grande de Tierra del Fuego. Tesis Doctoral, Facultad de Filosofía y Letras, Universidad de Buenos Aires.

-. 1989-1990. «Evolución cultural divergente en la Patagonia austral». Anales del Instituto de la Patagonia 19: 133-140.

-. 2003. «Taphonomy of the Tres Arroyos 1 Rockshelter, Tierra del Fuego, Chile». Quaternary International 109-110: 87-93. https://doi.org/10.1016/S1040-6182(02)00205-7

Borrero, Luis A., Fabiana M. Martin, Victoria D. Horwitz, Nora V. Franco, Cristian Favier Dubois, Florencia Borella, Flavia Carballo Marina, Juan B. Belardi, Patricia Campan, Ricardo Guichón, Sebastián Muñoz, Ramiro Barberena, Florencia Savanti y Karen Borrazzo. 2008. "Arqueología de la costa norte de Tierra del Fuego», en Arqueología de la costa patagónica. Perspectivas para la conservación, Isabel Cruz y Soledad Caracotche, eds., pp. 250-264. Río Gallegos: Universidad Nacional de la Patagonia Austral.

Bujalesky, Gustavo G. 1998. «Holocene coastal evolution of Tierra del Fuego». Quaternary of South America and Antarctic Peninsula 11: 247-282.

Calás, Elisa. 2014. «La subsistencia de los cazadores recolectores del Holoceno Medio y Tardío en el norte de Tierra del Fuego», en Cazadores de Mar y Tierra. Estudios recientes en arqueología fueguina, Jimena Oría y Angélica M. Tivoli, eds., pp. 171-195. Ushuaia: Editora Cultural Tierra del Fuego, Museo del Fin del Mundo.

Charlin, Judith, Karen Borrazzo y Marcelo Cardillo. 2013. «Exploring Size and Shape Variations in Late Holocene Projectile Points from Northern and Southern Coasts of Magellan Strait (South America)», en Mobility in Worldwide Prehistory and Early 
Complex Societies: Crossing Straits and Channels, François Djinjian y Janusz Koslowsky, eds., pp. 39-50. BAR International Series 2541. Oxford: Archaeopress.

Christensen, Marianne. 2016. La industria ósea de los cazadores-recolectores: el caso de los nómades marinos de Patagonia y Tierra del Fuego Chile. Punta Arenas: Universidad de Magallanes.

De Angelis, Hernán. 2015. Arqueología de los cazadores-recolectores de la faja central de la isla Grande de Tierra del Fuego. Buenos Aires: Sociedad Argentina de Antropología.

Estévez, Jordi y Ermengol Gassiot. 2002. «El cambio en sociedades cazadoras litorales: tres casos comparativos». Revista Atlántica-Mediterránea de Prehistoria y Arqueología Social 5: 43-85.

Fernández, Marilen, Juan F. Ponce, Francisco J. Zangrando, Ana M. Borromei, Lorena L. Musotto, Daniela Alunni y Martín Vázquez. 2020. «Relationships between Terrestrial Animal Exploitation, Marine Hunter-Gatherers and Palaeoenvironmental Conditions during the Middle-Late Holocene in the Beagle Channel Region (Tierra del Fuego)». Quaternary International 549: 208-217. https://doi.org/10.1016/j. quaint.2018.05.032.

Fiore, Danae.1999. «Diseños y técnicas en la decoración de artefactos: el caso de los sitios del Canal Beagle, Tierra del Fuego», en Actas del XIII Congreso Nacional de Arqueología Argentina, Tomo 2, pp. 75-89. Córdoba: Brujas.

Horwitz, Victoria y Marcelo Weissel. 2011. «Arqueología de Isla de los Estados; la frontera de la abundancia», en Los cazadores-recolectores del extremo oriental fueguino. Arqueología de Península Mitre e Isla de los Estados, Atilio F. Zangrando, Martín Vázquez y Augusto Tessone, eds., pp. 143-170. Buenos Aires: Sociedad Argentina de Antropología.

Isla, Federico y Gustavo Bujalesky. 2008. «Coastal Geology and Morphology of Patagonia and the Fuegian Archipelago». Developments in Quaternary Sciences 11: 227-239.

Kochi, Sayuri. 2017. «Paleodietas en cazadores-recolectores del canal Beagle durante el Holoceno tardío». Intersecciones en Antropología 18: 329-339.

Lanata, Jose Luis. 1995. Paisajes Arqueológicos y Propiedades del Registro en el Sudeste Fueguino. Tesis Doctoral, Facultad de Filosofía y Letras, Universidad de Buenos Aires.

Legoupil, Dominique. 1997. Bahía Colorada (île d'Englefield). Les premiers chasseurs de mammifères marins de Patagonie australe. Mémoires de l'A.D.P.F. París: Recherches sur les Civilisations.

- 2000. «El Sistema socioeconómico de los nómades del mar de Skyring (Archipiélago de Patagonia)». Anales del Instituto Patagonia 28: 81-119.

Legoupil, Dominique, Marianne Christensen y Flavia Morello. 2011. «Una encrucijada de caminos: el poblamiento de la isla Dawson (estrecho de Magallanes)». Magallania 39 (2): 137-152. http://dx.doi.org/10.4067/S0718-22442011000200010.

Mansur, Estela y Raquel Piqué, eds. 2012. Arqueología del Hain. Investigaciones etnoarqueológicas en un sitio ceremonial de la sociedad selknam de Tierra del Fuego. Madrid: Consejo Superior de Investigaciones Científicas.

Martin, Fabiana M. 2004. «Tendencias tafonómicas en el registro óseo humano del Norte de Tierra del Fuego», en Arqueología del Norte de la Isla Grande de Tierra del Fuego, Luis A. Borrero y Ramiro Barberena, eds., pp. 107-133. Buenos Aires: Dunken.

- 2006. Carnívoros y huesos humanos de Fuego-Patagonia. Aportes desde la Tafonomía Forense. Buenos Aires: Sociedad Argentina de Antropología.

Martin, Fabiana M., Mauricio Massone, Alfredo Prieto y Pedro Cardenas. 2009. «Presencia de Rheidae en Tierra del Fuego durante la transición Pleistoceno-Holoceno. Implicancias biogeográficas y paleoecológicas». Magallania 37 (1): 173-177. http://dx.doi.org/10.4067/ S0718-22442009000100014 
Massone, Mauricio. 2004. Los cazadores después del hielo. Santiago: Centro Diego Barros Arana.

-. 2009. Los cazadores del viento. Selk'nam. Santiago: Editorial Turismo Chile.

Massone, Mauricio, Donald Jackson y Alfredo Prieto. 1993. Perspectiva arqueológica de los selk'nam. Santiago: Centro Diego Barros Arana.

McCulloch, Robert D. y Flavia Morello. 2009. «Evidencia glacial y paleoecología de ambientes tardiglaciales y del Holoceno Temprano. Implicaciones para el poblamiento temprano de Tierra del Fuego», en Arqueología de la Patagonia, Mónica Salemme, Fernando Santiago, Myriam Álvarez, Ernesto Piana, Martín Vázquez y Estela Mansur, eds., pp. 119-139. Ushuaia: Utopías.

McCulloch, Robert D., Mike J. Bentley, Ross S. Purves, Nick R. J. Hulton, David E. Sugden y Chalmers M. Clapperton. 2000. «Climatic Inferences from Glacial and Palaeoecological Evidence at the Last Glacial Termination, Southern South America». Journal of Quaternary Science 15 (4): 409-417.

Montes, Alejandro y Daniel Martinioni. 2017. «Geomorfología y paleoambientes Holocenos de la costa atlántica de Tierra del Fuego entre el Río Grande y el estrecho de Le Maire», en Patrimonio a orillas del mar. Arqueología del Litoral Atlántico de Tierra del Fuego, Martín Vázquez, Dolores Elkin y Jimena Oría, eds., pp. 29-44. Ushuaia: Editora Cultural Tierra del Fuego.

Morello, Flavia, Lino Contreras y Manuel San Román. 1999. «La localidad de Marazzi y el sitio arqueológico Marazzi I, Una re-evaluación». Anales del Instituto de la Patagonia 27: 183-197.

Morello, Flavia, Manuel San Román y Alfredo Prieto. 2004. «Obsidiana verde en FuegoPatagonia: distribución y estrategias tecnológicas», en Contra viento y marea. Arqueología de Patagonia, María T. Civalero, Pablo Fernández y Gabriela Guraieb, eds., pp. 149-166. Buenos Aires: INAPL-SAA.

Morello, Flavia, Luis A. Borrero, Mauricio Massone, Charles Stern, Arlene Garcia-Herbst, Robert McCulloch, Manuel Arroyo Kalin, Elisa Calas, Jimena Torres, Alfredo Prieto, Ismael Martínez, Gabriel Bahamonde y Pedro Cárdenas. 2012. «Hunter-Gatherers, Biogeographic Barriers and the Development of Human Settlement in Tierra del Fuego». Antiquity 86 (331): 71-87.

Muñoz, Andrés S. 1997. «Explotación y procesamiento de ungulados en Patagonia meridional y Tierra del Fuego». Anales del Instituto de la Patagonia 25: 201-222.

Muñoz, Andrés S. y Juan Bautista Belardi. 2011. «Nueva información sobre viejos datos: arqueología del norte de Peninsula Mitre», en Los cazadores-recolectores del extremo oriental fueguino. Arqueología de Península Mitre e Isla de los Estados, Atilio F. Zangrando, Martín Vázquez y Augusto Tessone, eds., pp. 171-202. Buenos Aires: Sociedad Argentina de Antropología.

Ocampo, Carlos, Pilar Rivas y Eugenio Aspillaga. 2000. «Chenke en Bahía Felipe, cost noroccidental de Tierra del Fuego». Anales del Instituto de la Patagonia 28: 215-223.

Oría, Jimena y Florencia Mari. 2019. «Resolución temporal y espacial en el sitio arqueológico Yowen Ko, laguna Amalia (Tierra del Fuego)», en Arqueología de la Patagonia. El pasado en las arenas, Julieta Gómez Otero, Ariadna Svoboda y Anahí Banegas, eds., pp. 409-420. Puerto Madryn: Instituto de Diversidad y Evolución Austral.

Oría, Jimena y Mónica C. Salemme. 2019. "Shallow Lake Environments in the HunterGatherer's Way of Life (Tierra del Fuego, Argentina)». Latin American Antiquity 30 (1): 109-126. https://doi.org/10.1017/laq.2018.67 
Oría, Jimena, Mónica C. Salemme, Martín Vázquez, Valeria Bártoli y Ramiro López. 2021. «Surface Site Formation Processes in Northern Tierra del Fuego (Argentina): A Case Study of the Amalia 4 Site». Geoarchaeology 36 (1): 18-33.

Orquera, Luis A. y Ernesto L. Piana. 1988. «Human Littoral Adaptation in the Beagle Channel Region: The Maximum Posslble Age». Quaternary of South America and Antarctic Peninsula 5: 133-165.

-. 1999. Arqueología de la región del canal Beagle (Tierra del Fuego, República Argentina). Buenos Aires: Sociedad Argentina de Antropología.

-. 2006. «El poblamiento inicial del área sudamericana sudoccidental». Magallania 34 (2): 21-36. http://dx.doi.org/10.4067/S0718-22442006000200003

Piana, Ernesto L., Augusto Tessone y Atilio F. Zangrando. 2006. «Contextos mortuorios en la región del Canal Beagle: del hallazgo fortuito a la búsqueda sistemática». Magallania 34 (1): 87-101. http://dx.doi.org/10.4067/S0718-22442006000100007

Piana, Ernesto L., Atilio F. Zangrando y Luis. A. Orquera. 2012. «Early Occupations in Tierra del Fuego and the Evidences from S Layer in Imiwaia I Site (Beagle Channel, Argentina)», en Southbound: Late Pleistocene Peopling of Latin America, Laura Miotti, Mónica C. Salemme, Nora Flegenheimer y Ted Goebel, eds., pp 171-175. College Station: Center for the Study of the First Americans.

Ponce, Juan F., Ana Borromei y Jorge O. Rabassa. 2011. «Evolución del paisaje y de la vegetación durante el Cenozoico tardío en el extremo sudeste del archipiélago Fueguino y Canal Beagle», en Los cazadores-recolectores del extremo oriental fueguino. Arqueología de Península Mitre e Isla de los Estados, Atilio F. Zangrando, Martin Vázquez y Augusto Tessone, eds., pp. 51-63. Buenos Aires: Sociedad Argentina de Antropología.

Prieto, Alfredo, Susana Morano, Pedro Cárdenas, Victor Sierpe, Elisa Calas, Marianne Christensen, Christine Lefevre, Veronique Laroulandie, Yolanda Espinosa-Parrilla, Oscar Ramirez, Carles Lalueza-Fox, Erika Hagelberg, Rodrigo Cárdenas y Jorge Gibbons. 2020. «A Novel Child Burial from Tierra del Fuego: A Preliminary Report». The Journal of Island and Coastal Archaeology 15 (3): 436-454. https://doi.org/10.1080/15564894.2019.1643429.

Rabassa, Jorge, Andrea Coronato, Gustavo Bujalesky, Mónica C. Salemme, Claudio Roig, Andrés Meglioli, Calvin Heusser, Sandra Gordillo, Fidel Roig, Ana Borromei y Mirta Quattrocchio. 2000. «Quaternary of Tierra del Fuego, Southernmost South America: An Updated Review». Quaternary International 68-71: 217-240. https://doi.org/10.1016/ S1040-6182(00)00046-X

Salemme, Mónica, Gustavo Bujalesky y Fernando Santiago. 2007a. «La Arcillosa 2: la ocupación humana durante el Holoceno Medio en el Río Chico, Tierra del Fuego, Argentina», en Arqueología de Fuego-Patagonia. Levantando piedras, desenterrando huesos ... y develando arcanos, Flavia Morello, Mateo Martinic, Alfredo Prieto y Gabriel Bahamonde, eds., pp. 723-736. Punta Arenas: CEQUA.

Salemme, Mónica, Fernando Santiago, Jorge A. Suby y Ricardo Guichón. 2007b. «Arqueología funeraria en el norte de Tierra del Fuego». Actas del XVI Congreso Nacional de Arqueología Argentina. Tras las Huellas de la Materialidad, pp. 71-77. San Salvador de Jujuy: Universidad Nacional de Jujuy.

San Román, Manuel. 2014. «Sea-Level Changes and Coastal Peopling in Southernmost Pacific South America: Marine Hunters from Patagonia», en Encyclopedia of Global Archaeology, Claire Smith, ed., pp. 6515-6525. Nueva York: Springer-Verlag.

San Román, Manuel. 2016. Stratégies économiques et sociales des chasseurs marins de Patagonie: Archéozoologie des sites anciens du détroit de Magellan et des mers intérieures. Tesis Doctoral. Paris: L’Université de Paris I, Panthéon-Sorbonne. 
San Román, Manuel, Flavia Morello y Alfredo Prieto. 2002. «Nuevos antecedentes sobre explotación de recursos faunísticos en el mar de Otway y canales adyacentes». Anales del Instituto de la Patagonia 30: 147-154.

Santiago, Fernando, Mónica C. Salemme, Jorge Suby y Ricardo Guichón. 2011. «Restos humanos en el norte de Tierra del Fuego: Aspectos contextuales, dietarios y paleopatológicos». Intersecciones en Antropología 12: 147-162.

Santiago, Fernando y Mónica C. Salemme 2016. "Guanaco Hunting Strategies in the Northern Plains of Tierra del Fuego, Argentina». Journal of Anthropological Archaeology 43: 110-127. https://doi.org/10.1016/j.jaa.2016.07.002

Scheinsohn, Vivian. 1993/1994. «Hacia un modelo del aprovechamiento de las materias primas óseas en la isla Grande de Tierra del Fuego (Argentina)». Relaciones de la Sociedad Argentina de Antropología XIX: 307-324.

-. 2014. "Bone Material and Design Choices in Southern Patagonia", en From These Bare Bones: Raw Materials and the Study of Worked Osseous Objects, Alice Choyke y Sue O’Connor, eds., pp.36-49. Oxford: Oxbow Books.

Tessone Augusto, Atilio F. Zangrando, Susana Valencio y Héctor Panarello. 2003. «Isótopos estables del carbono en restos óseos humanos de la región del Canal Beagle, Isla Grande de Tierra del Fuego». Revista Argentina de Antropología Biológica 5 (2): 33-43.

Tessone, Augusto, Ricardo Guichon, Jorge Suby y Livia Kozameh. 2011. «Bioarqueología de Península Mitre», en Los cazadores-recolectores del extremo oriental fueguino. Arqueología de Península Mitre e Isla de los Estados, Atilio F. Zangrando, Martín Vázquez y Augusto Tessone, eds., pp. 231-270. Buenos Aires: Sociedad Argentina de Antropología.

Tivoli, Angelica M. 2010. Las aves en la organización socioeconómica de cazadoresrecolectores-pescadores del extremo sur sudamericano. Tesis Doctoral, Facultad de Filosofía y Letras, Universidad de Buenos Aires.

Vázquez, Martín. 2019. «Distribución del registro arqueológico en la costa norte de Península Mitre, Tierra del Fuego», en Arqueología de la Patagonia: el pasado en las arenas, Julieta Gómez Otero, Ariadna Svoboda y Anahí Banegas, eds., pp 181-192. Buenos Aires: CONICET-IDEAUS.

Vázquez, Martín, Atilio F. Zangrando, Augusto Tessone y Antonio Ceraso. 2011. «Arqueología de la costa meridional de Península Mitre», en Los cazadores-recolectores del extremo oriental fueguino. Arqueología de Península Mitre e Isla de los Estados, Atilio F. Zangrando, Martín Vázquez y Augusto Tessone, eds., pp. 203-229. Buenos Aires: Sociedad Argentina de Antropología.

Vidal, Hernán J. 1985. Los conchales de Bahía Valentín. Tesis de Licenciatura. Facultad de Filosofía y Letras, Universidad de Buenos Aires.

Zangrando, Atilio Francisco. 2009. Historia evolutiva y subsistencia de cazadores-recolectores maritimos de Tierra del Fuego. Buenos Aires: Sociedad Argentina de Antropología.

Zangrando, Atilio F., Augusto Tessone y Martín Vázquez. 2009. «El uso de espacios marginales en el archipiélago fueguino: implicaciones de la evidencia arqueológica de bahía Valentín», en Arqueología de la Patagonia. Una mirada desde el último confín, Mónica C. Salemme, Fernando Santiago, Myriam Álvarez, Ernesto Piana, Martín Vázquez y Estela Mansur, pp. 47-62. Ushuaia: Utopías.

Zangrando, Atilio F., Hein B. Bjerck, Ernesto L. Piana, Heidi M. Breivik, Angélica M. Tivoli y Joan Negre. 2018. «Spatial Patterning and Occupation Dynamics during the Early Holocene in an Archaeological Site from the South Coast of Tierra del Fuego: Binushmuka I». Estudios Atacameños 60: 31-49. 